\title{
A Review of Misinformation Across Disciplines: Implica- tion for Online Education
}

\author{
Ting Huang
}

Corresponding author contact

Ting Huang,

College of William and Mary, 301 Monticello Avenue,

Williamsburg, VA 23185

Orcid ID: https://orcid.org/0000-0003-2883-9230

thuang03@wm.edu

\begin{abstract}
Misinformation research has grown to become a critical topic in all disciplines. Since the expanding of online media, misinformation has been spreading rapidly across the globe through social media and other information systems. Paralleling the rise of academic interest in misinformation, is the emergence of online education scholarship. Interest in the online educational implications of misinformation and its impact attracts an increase in scholarship on misinformation. This article presents the results of a review of 1172 publications with "Misinformation" across disciplines and a subset of 174 misinformation literature in online education that were published between 2009 and 2021. This review answers three questions: (1) What is the overall distribution of publication activity with "misinformation" publications? (2) What methodologies have scholars used to investigate misinformation involving online education? (3) What have scholars reported about the results of studies involving misinformation in online education? The review reveals that various methodologies were used in literature focusing on misinformation online education with leading numbers of content analysis and quantitative studies. This systematic review is particularly relevant to those online educators in various disciplines who are interested in learning what scholars from their own academic disciplines are writing about misinformation.
\end{abstract}

Keywords: review, misinformation, online education, research, discipline

\section{Background}

The wide and fast spread of misinformation online raised many concerns. Before COVID, many studies on misinformation were generated from the cognitive, psychological, communicative, health information, and many other fields. In election years (e.g., around 2016), political misinformation has detrimental effects on democracy. Between 2019 to present, misinformation attracts increasingly intensifying research attention to COVID and vaccine topics. Not only misinformation literature is blooming in all disciplines, misinformation scholarship also has largely grown to health field, political science, and many fields [30]. 


\section{Introduction}

Misinformation has drawing attention from online educators in various fields with heavy discussions in social media and impacts of misinformation [6, 43]. Because misinformation online is a relative new field which emerged in recent decade with fastest growth last year in 2020, research on misinformation education is scare and is still developing[30]. Misinformation has devastating consequences in all disciplines. It can influence people's decisions that negatively impact their emotions, healthcare choices, positive life attitudes, and personal well-beings [45, 46]. For example, if cancer patients were provided misinformation about their cancer treatment choices, misinformation could be negatively impacting their good path of treatment and sometime this kind of misinformation could be fatal [55].

Misinformation is one of the major challenges in an era of drastically developing online education because the educational priorities in the 21st century include abilities to "identify false statements and fallacious reasoning" ([39], p, 40). Due to the expanding landscape of online education, educators have a critical goal to develop students' digital literacy skills in crucially evaluate information [27]. There are vastly increased opportunities for circulation of misinformation for online education, making students exposure to misinformation, alternative facts, fake news, disinformation, so much common. It is also hard to provide corrective actions to the misinformation due to the continuously advancement of online media $[33,47]$. Because misinformation online is a relative new field which emerged in recent decade with fastest growth last year in 2020, research on misinformation education is scare and is still developing.

\section{Review of Related Literature and Method}

\subsection{Review of Related Literature}

The field of misinformation is growing. Misinformation research has been growing rapidly on topics such as COVID 19 [2, 4, 48], vaccine [6; 9, 30, 49], climate change [13, 16, 36], medical concerns [56], communication [8], memory [22], cognitive impacts on reading [45], new literacies [11], online education [9], political studies $[11,1]$, and diversity [42] etc.

In social science and medical field, there are already serious concerns about the negative impact of what we read, hear and see on social media (such as Facebook, twitter, Instagram, Pinterest, TikTok as discussed in the result section). Some of these types of misinformation was driven by political agenda, others were may be circulated by accidents or even in sarcastic ways. Sometimes, each information found in research studies could contain misinformation due to the ease of online publishing and the growing social media landscape [45]. Whether watching news programs, perusing blogs, viewing videos on YouTube, students have been exposed to a mix of scientific information, evidence-based opinions, data-driven partial truths, unknowable conjectures, uninformed claims, blatant fake news, alternative facts, propagandas, and even purposeful lies. Concerns about the accuracy of multimodal misinformation in texts, voices, and videos had driven drastically increased research attentions. Some misinformation was largely driven by the pandemic, vaccines, health information concerns, political disputes, criticisms of journalists and news outlets, the ease of online publishing, trolling behavior, the social media landscape, and a host of other contemporary considerations and practices 
$[1,15,29,33]$. As outlined by [34], these concerns have intensified interest in articulating the factors related to, results of, and mechanisms underlying experiences with misinformation.

On an educational viewpoint, misinformation could impact positive democratic decision making and disable societal improvement in a more just and productive democracy [29]. In the educational research field, the bulk of misinformation research was heavily done in the psychological and cognitive tradition of studying misinformation effects. For example, how misinformation impacted reading comprehension and retention. This scholarship of educational cognitive studies commonly used experimental designs of eliminating misinformation effects. For example, [15] found that refutation texts that elaborate inaccurate concepts could be effective in helping readers overcome previous held misunderstanding about STEM topics in physics principles and climate change. [45] found similar refutation texts could help with historical civil rights misconceptions for readers.

Educational research also had contributed to misinformation field, even though these lines of misinformation research may not necessarily focus on online education implications. Research in education has demonstrates the facets in, results of, and impacts by misinformation. For example, in the field of literacy/reading research in education, research has proven that misinformation on texts have long term negative impact on reading. For another example, [17] and [35] found that individuals generally have reluctance to completely disregard previously acquired knowledge even if this held knowledge is misinformation [17, 35]. Based on knowledge norms [51], many people are unlikely to know that Marie Curie was the scientist who discovered radium. In an experimental design, [37] found that participants who prompted with misinformation reading claiming that Pasteur discovered radium, they might answer the question "What is the name of scientist that discovered radium?" with the false information, which is Pasteur was the one discovered radium [37]. Worse, participants may also reproduce false information they already know is wrong. Generally, people are aware that the pilgrims traveled to America on the Mayflower. After reading a statement indicating the ship was the Godspeed, some participants nevertheless answer related questions (e.g., "What ship brought the pilgrims to America?") with the incorrect lure. The negative consequences of being presented with "alternative facts" obtain even when participants should have existing knowledge about what is and is not correct.

This review focuses on misinformation field in all disciplines and then drill down to discuss the research implications in the field of online education. This review provides a picture of what we know about misinformation across disciplines and how this work has an implication for online education. In the first section, I focus on data analysis of 1172 article abstracts that I searched and found between 2009-2021 with the key words of "misinformation" via Scopus. Next, I analyzed the misinformation literature of 1172 publications across disciplines. Then, I discuss the methodologies used in a sample of 174 publications of misinformation related to online education before I offer a conclusion of the general results in this work. In this review, I answer the following research questions:

(1) What is the overall distribution of publication activity with "misinformation" in the 1172 publications? 
(2) What methodologies have scholars used to investigate misinformation involving online education in the 174 publications as the sample of literature containing "misinformation" and "online education"?

(3) What have scholars reported about the results of studies involving misinformation in a sample of literature containing "misinformation" and "online education"?

\subsection{Method}

The initial search yields a comprehensive collection of scholarly literature on the topic of misinformation. I started with searching on Scopus. I used Scopus because it has a comprehensive collection of over 34,346 various disciplinary research databases, indexes, and topic-specific online resources1. I reviewed Scopus to generate a list of peer-reviewed journal articles and conference papers on misinformation. Scopus also allowed me for advanced searches in titles within time periods. Specifically, during the review, Scopus were searched three times in 2021 during the months of January, March, and April, yielding similar results. I also searched on google scholar, and other available databases in my current institutional library (such as JSTOR and ERIC). I also combined these searches with snowball searching. For instance, if I found one particular article discusses misinformation with an online education focus, I will scan through its references and find relevant articles to read.

I then set up with parameters for limiting the boundaries of the search. The scope was restricted to peer-reviewed journal articles and conference papers published from the blooming of misinformation research from 2009 through the 2021 when the review was being conducted. This is because based on research on Scopus, before 2009, the number of studies containing "misinformation" were less 20 annually. From the period of 1912-1991, the number of studies containing "misinformation" were less 10 annually. It makes sense to start with 2009 and focusing on the most recent decade up until the time of this writing moment (April, 2021) because this is the period when the number of articles on misinformation increased drastically, see Figure 1 for the trend.

Figure 1

Number of Articles (Documents) with "Misinformation" in the Title from 2009-2021

1 Scopus (Elseriver) is the largest abstract and citation database of peer-reviewed literature: scientific journals, books and conference proceedings. Scopus is Elsevier's abstract and citation database launched in 2004. Scopus covers nearly 36,377 titles (22,794 active titles and 13,583 inactive titles) from approximately 11,678 publishers, of which 34,346 are peer-reviewed journals in top-level subject fields. Delivering a comprehensive overview of the world's research output in the fields of science, technology, medicine, social sciences, and arts and humanities, Scopus features smart tools to track, analyze and visualize research. 


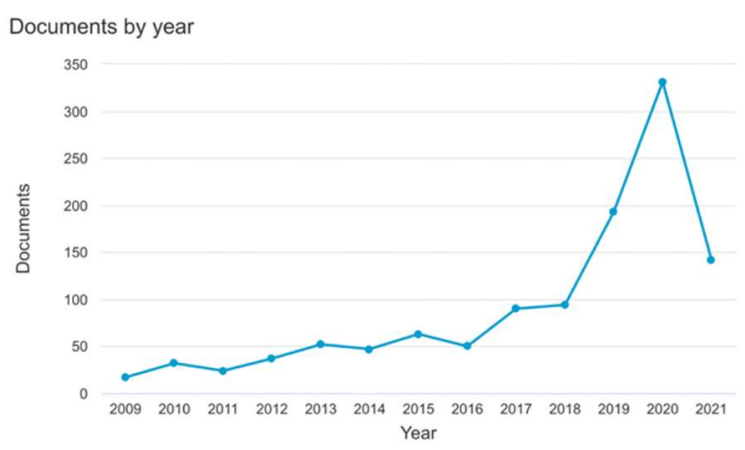

After the scope is set with years between 2009-present (i.e., April 2021) with key word "misinformation", I downloaded the data set in CSV form and ris files for analysis. Next, for the 1172 articles to offer a clear picture about misinformation across disciplines, I used a data visualization tool VOSviewers to demonstrate the density of topical words via content analysis using colored visual connections. The larger the knot, the higher frequency the wording appeared in the 1172 articles. Within the 1172 misinformation articles and conference proceedings, I wanted to further narrow down to key words "online education". The bibliography was downsizing to 210 articles and papers. The complete alphabetized bibliography of the final sample of 210 peer-reviewed articles and papers with "Misinformation" and "Online Education" in the title were exported from Scopus in an CSV form. These 210 articles were further downsizing to 174 articles due to software errors, duplications/repetitions, and missing information on abstracts. Lastly, I further transformed CSV form into Excel and manually coded all these 174 articles. Fulltext copies of every article and paper in the bibliography were obtained so that they could be read and categorized by (1) discipline area as defined by the National Center for Education Statistics (2021), (2) research methodologies of studying misinformation in online education, and (3) results of misinformation research.

This systematic review hopes to contribute to the misinformation field by addressing an existing gap in online education. One of the primary differences between the existing reviews on misinformation and this review of misinformation is the scope of content included. This review includes 1172 publications and a subset of 174 misinformation online education articles, which is the largest volume on misinformation multidiscipline review. In addition, there are differences between this review and other existing reviews on the foci of misinformation. The existing reviews [2, 50; 54] include a collection of disciplinary specific misinformation research related to COVID or health science fields (e.g., [31] reviewed health misinformation online), may or may not include online education as their foci, may or may not write "misinformation" in their title, may or may not review the most recent literature of misinformation including pandemic explorative scope of misinformation research, or may or may not discuss misinformation from an online education viewpoint (e.g., [2] reviewed COVID misinformation but not with an educational foci).

\section{Results}


The results are presented to correspond with the order of the research questions. Each question is restated and data from the sample of misinformation literature is provided to answer each question.

\subsection{Overall Distribution of Publication}

The first question was: What is the overall distribution of publication activity with "misinformation" in the 1172 publications?

The sample of literature included 1172 journal articles and conference papers written in various disciplines. This section include discussion on the key content found using data visualization software VOSviewer. Figure 2 shows education related misinformation topics in the 1172 article abstracts found via data from Elseriver Scopus. The visualization was analyzed via VOSviewer software.

\section{Figure 2}

The Visualization of Key Words Appeared in the Title of 1172 Publication with "Misinformation" Published during 2009-2021 Analyzed via VOSviewer

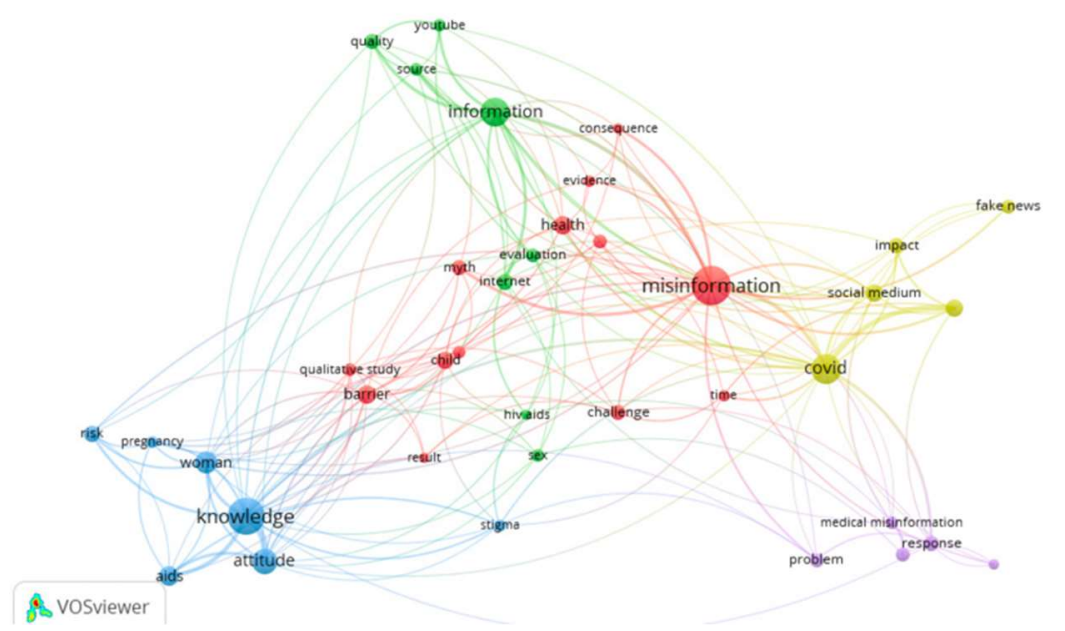

From Figure 2, we can see with "misinformation" being the centered focus, there are a few stations of sub foci: covid, social medium, response, medical misinformation, problem, challenge, internet, information, knowledge, woman, attitude, pregnancy, barrier, child, myth, internet, health, evidence...The same color lines mean connections among the studies. The size of the color ball means the volumes of publication counts. 
These items fell outside of the parameters defined for the present systematic review, which was designed to examine only misinformation publications that were clearly identified through the title as having a focus. However, some usable articles and papers were found in the existing reviews of misinformation [2, 50,54] and these items extended the sample beyond those publications obtained through the database searches.

In terms of trend of growth on publications of misinformation, the documents per year by publication sources were demonstrated by Figure 3. As shown in Figure 3, from 2014 to 2017, the Journal of Applied Research in Memory and Cognition has published the highest numbers of articles on misinformation. From 2019 to present, the American Journal of Public Health has published the largest quantity of journal articles on misinformation. Since 2014, open access PLOS One also has a visible line of publishing on misinformation.

Figure 3

Sources that Publishing on Misinformation, Numbers Per Year and Trend.

\section{Documents per year by source}

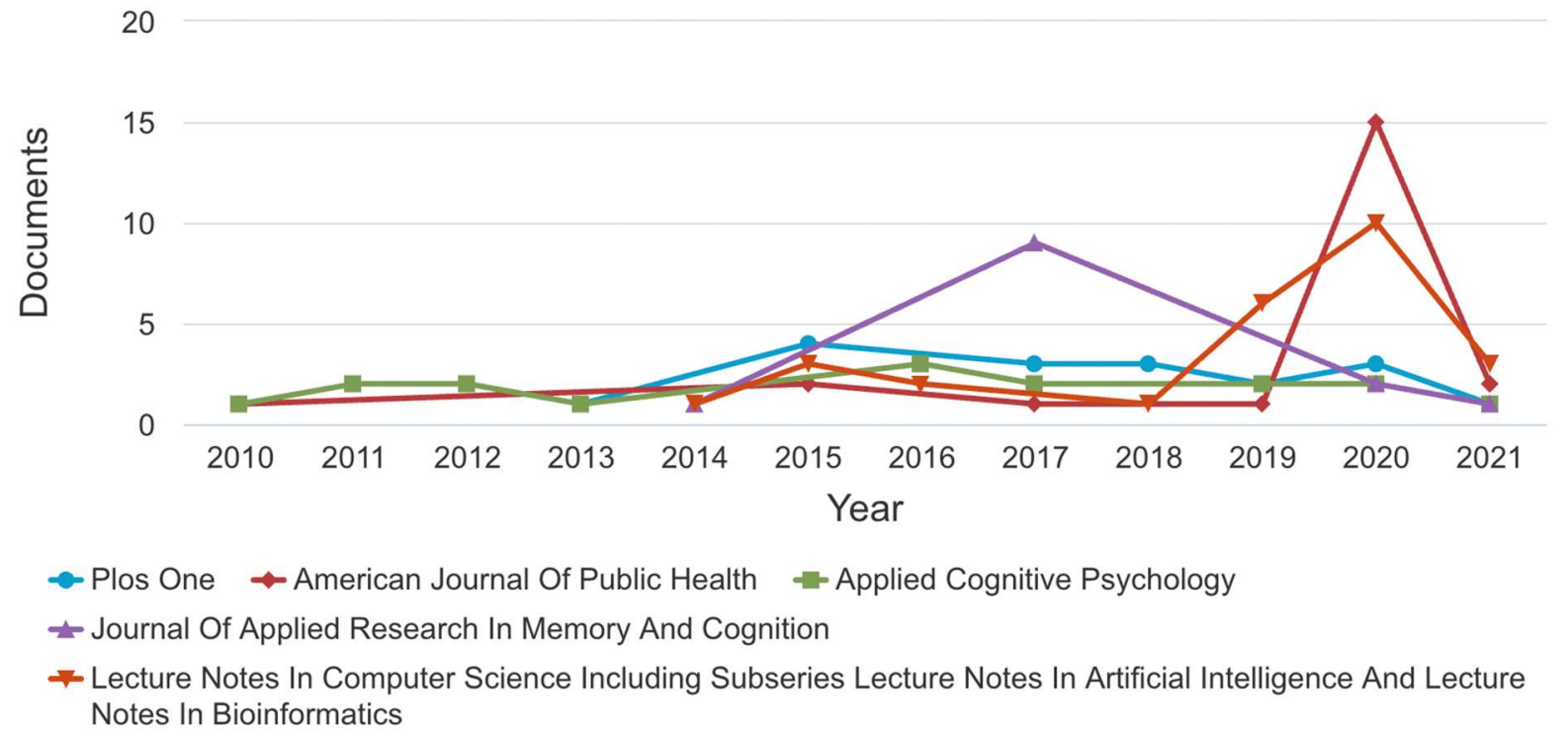




\subsection{Methodologies in Misinformation and Online Education}

The second question was: What methodologies have scholars used to investigate misinformation involving online education in the 174 publications as the sub-set sample of literature containing "misinformation" and "online education"?

Within the 1172 misinformation publications about online education, I narrowed down the collection of publications to 174 by searching publications that contain the wording "online education" in the title and/or abstract, and then data cleaning as mentioned in the prior method section. As mentioned, after data coding of methodology using an Excel form for these 210 articles and the usage of pivotal tables, I deleted 7 repeated titles, 6 articles that lacked abstract information, 3 repetitions. That leaves me with 174 articles. Among the remaining 174 articles with "misinformation" and "online education" as the focus (appeared in their title and/or abstract), below are the distributions of their methodologies as demonstrated in Table 1. Publications from the field of Education of Health took the lead in terms of instructional methodologies for misinformation, although several other discipline areas included teaching-related articles or papers, most notably Social Media related Information Education.

In terms of methodology, the largest category of publication is Empirical Content analysis (E-Content analysis), which included one fourth of the 174 publications. This category, which has 43 publications, presented lists of general techniques of using existing online media data for analyzing implication of misinformation for online education in various disciplines such as topics on pandemic, cancer, vaccine, nutrition, smoking, political attitudes, reading comprehension, communication patterns, and trends of misinformation. Content analysis here are further divided into sub-categories, such as social network analysis, evaluating content, evaluating quality, content/text analysis, and sentiment analysis. It is worth noting that in this category of publication, there are prominent attention paid to social media. Details of these studies will be discussed in next section in the third research question.

Empirical Quantitative Experiment studies (E-Quant-Experiment) was the second largest group, covering research in typical effects of misinformation in a pre- and postexperiments designs using experimental and controlled conditions. Conceptual Papers fell in third place, with various disciplines that discussed both general and discipline-specific strategies for combating misinformation as supplementary online education perspectives. These top three categories comprise $64 \%$ of the 174 publications discussing misinformation and online education, see Table 1. 
Table 1

Methodologies Represented in the Sample of 174 Articles and Papers

\begin{tabular}{|c|c|c|}
\hline $\begin{array}{c}\text { Methodologies Employed } \\
\text { in the Paper }\end{array}$ & Counts & Notes \\
\hline $\begin{array}{c}\text { E-Content Analysis (e.g., } \\
\text { databases, website hits, } \\
\text { videos) }\end{array}$ & 43 & $\begin{array}{c}\text { Content analysis across different methods, } \\
\text { such as using videos for analysis, using } \\
\text { websites (Google, Yahoo, or a Patient Q\&A } \\
\text { service portal data), or databases to } \\
\text { understanding factors in and relations among } \\
\text { misinformation, online education, and social } \\
\text { media. }\end{array}$ \\
\hline E-Quant-Experiment & 36 & $\begin{array}{c}\text { Most had a pre- and post-tests designs to } \\
\text { understand impact of misinformation in various } \\
\text { disciplines. Others using online portals collecting } \\
\text { data for experiments related to effects of } \\
\text { misinformation. }\end{array}$ \\
\hline Conceptual Paper & 33 & $\begin{array}{c}\text { Conceptual paper in various disciplines, some } \\
\text { are calling for change, others provide framework, } \\
\text { still others provide a research agenda, some } \\
\text { strategies, a pedagogy, a model for combating } \\
\text { misinformation. }\end{array}$ \\
\hline $\begin{array}{l}\text { E-Quant-Survey (e.g., } \\
\text { online or cross sectional, } \\
\text { regression modeling) }\end{array}$ & 26 & $\begin{array}{c}\text { Survey methods are common, even though some } \\
\text { studies are larger scale such as cross sectional, } \\
\text { multi-countries, while others using online } \\
\text { collected data for SPSS analysis such as } \\
\text { regression modeling for factors related to } \\
\text { misinformation in various disciplines (Health } \\
\text { science, cognitive studies, psychology, political } \\
\text { science...) }\end{array}$ \\
\hline $\begin{array}{c}\text { Review (Calling for change, } \\
\text { meta-analysis) }\end{array}$ & 16 & $\begin{array}{l}\text { Review papers vary in the scales, there are } \\
\text { papers using a sample of } 69,57, \text { or } 32 \text { articles/or } \\
\text { abstracts, while there are reviews using massive } \\
\text { publications such as } 5467 \text { articles. However, none } \\
\text { of these reviews focus on misinformation online } \\
\text { education across disciplines. Many of these }\end{array}$ \\
\hline
\end{tabular}


reviews focus on a particular discipline such as health information or COVID.

E-Qualitative (focus groups, interviews, case studies, grounded theory)

E-mixed method

Introduction a program

Case study; E-quant-survey

E-Survey, E-Conceptual Model

Pedagogical Conceptual

Framework-Verifi2, with E interviews

Program evaluations
13

2

2

1

1

1

(n)

\begin{abstract}
Qualitative studies are much less in the misinformation literature. Mostly using interviews or semi-structured interviews.
\end{abstract}

Mixed methods studies are even rare than qualitative designs in misinformation literature with online education.

There are two articles found to propose a program to provide corrective strategies for misinformation.

This particular study used a combination of case study and quantitative survey analysis.

This particular study used a combination of conceptual modeling and quantitative survey analysis.

This paper is from computer science background, which they propose a digital program called Verifi2 for combating misinformation. They also explored the programs with users via interviews.

There is one study about assessment of a program.

\subsection{Results of Misinformation and Online Education}

The third question was: What have scholars reported about the results of studies involving misinformation in a sample of literature containing "misinformation" and "online education"?

The result shows that scholars in the field of misinformation and online education had focused on social media and its impact on misinformation in various positive and negatives effects. Some studies demonstrated the importance of social media played in educating people about misinformation. For example, flagging misinformation on social media is an important strategy to help people discern misinformation [32]. Social media per se can support combatting vaccine misinformation by implementing standards to educate people [49]. In this sample 174 publication on misinformation and online education, there are 50 articles chose to focus on social media. Some articles generally discussed social media and may include more than two social media platforms [Allcott, Gentzkow, \& Yu, 
$2019,7]$. Other studies reported that social media is responsible for spreading misinformation [52]. Still other articles specifically investigated one type of social media, such as Twitter [52], Facebook [43], Pinterest [5], WhatApp [5], Instagram [38], as well as video analysis on TikTok (for 100 videos with \#covidvaccine, [6]) videos. There are also analysis using data on websites (e.g., Google) and online databases (e.g., Patient online Q\&A portal data). Only one study focused on YouTube medical videos [23], which creates space for future video learning and misinformation scholarship.

Second, the results of these articles collectively point to the idea of being able to critically evaluate misinformation and information online is one of the keys in getting succeed in digital literacy skills as a 21-century citizen. Online educators have argued that it is important to teach digital literacy and teach students to evaluation resources [12]. This aligns with K-12 professional standards, such as Common Core Standards asked students to evaluation resources [29].

Third, scholars in misinformation had worked on belief systems and how prior beliefs and experiences impacted their acceptance or rejection misinformation (e.g, private school girls and parents who did not get vaccine on HPV impacted their acceptance of HPV vaccine misinformation. Another example, gender(females), higher level of education, and age play roles in acceptor misinformation. Females accepted more misinformation than male.

Fourth, the reviewed studies also touched on the issues of diversity and misinformation. The results of these studies all point to that gender, race, class, age, ethnicity, and socioeconomic status also play roles in misinformation acceptance rate. For example, people of color accepted more misinformation than white people. [4, 42] offered an examination of factors contributing to the acceptance of online health misinformation. Lower socioeconomic status, older age, and lower educational level groups are also disproportionally impacted by misinformation [48]. It is worthy to mention that among the results found by this line of research, studies also have shown contradictory results. For example, some study argued that males tend to trust misinformation more than females [48], while other study contended that females accepted more health misinformation than their male counterparts [42].

Lastly, the recent misinformation studies in online education has discussed a list of strategies of combating misinformation and educating people about discerning misinformation. Strategies including social media being used as a way of combating misinformation. For example, flagging is effective means to use on social media platform. Another means social media can implement is to use standards and consult with professionals in evaluating misinformation. Additionally, studies call that misinformation scholars to speak up. This includes that, for example, pharmacists speak up when they see misinformation of health care among patients with emotional support so that people can overcome misinformation. For another example, health practitioners need to speak up and not keep silence so that patients even with prior biases can get informed decisions in scientific knowledge background. Some additional methods of combating misinformation include offering accurate accounts accompanied by supporting information [e.g., creditable source organizations, strong data support, and trustworthy of figures/celebrities promote the scientific information; [10], [18]). Still other studies examine what happens when people are presented with information that contradicts what they know or believe to be true.

In a word, all fields and disciplines of misinformation scholars had unanimously asked people to stay connected (the good and bad of social media in offering scientifically knowledge and spreading misinformation) and use their professional knowledge and emotional support to help combat misinformation [19, 37]. 


\section{Discussion and Limitation}

Misinformation is a serious issue in online education [9]. When students learn formally or informally nowadays, they are online searching and learning from information that feed to them. These learning activities include watching news, checking out YouTube, reading texts on videos, and conversing with others via on online media. They may be exposed to amix of misinformation and scientific knowledge. These information include informed opinions, partial truths, and even pure lies. When educators were trained in teaching preparation programs, the curriculum did not specifically address the issue of how to educate teachers about preparing students critically think about the information they access online information such as videos on YouTube because online education and online learning was just a very recent phenomenon in the recent three decades.

The analysis of 1172 of misinformation research and the subset of 174 publications of misinformation in online education provides evidence the growing importance of research fields across disciplines focused on the topic of misinformation. We can see the trend that misinformation called more and more attention due to the current pandemic, COVID, social justice issues in political world, and long-standing medical issues. Yet, we do not know the exact agenda of researchers, especially online educators in misinformation field who are studying misinformation or what their future goals might be. Some authors had a single publication, while others published multiple articles or papers. Future literature reviews could be conducted to reveal longitudinal trends, or the extent to which misinformation remains a focus of scholarly interest.

In terms of research methods, research that has been conducted on misinformation is somewhat varied and tends to be closely related to problems and issues inherent to the related discipline area. For example, the information technology studies delve into attributes of the social media technologies or user data that could fuel the defeating misinformation on these sites. However, several studies from multiple discipline areas investigated attributes of help combat misinformation [29], analysis of misinformation online [6], or a combination of both. There were 43 studies that used a basic content-analysis strategy involving the sharing of misinformation having the characteristic of interest, matching with personal background, echoing prior experiences, recording of observations, and final analysis where conclusions were made. In addition, qualitative research in misinformation and online education is limited.

This may be an indication that research methodologies for online misinformation spreading are not yet well defined or have not reached a level of sophistication that would permit more advanced approaches misinformation empirical research. Future work developing methodologies for online education and misinformation analysis, and related data (comments, user demographics, etc.) could lead to more advanced research paradigms and deeper understanding of misinformation sharing environments. Some work along these lines are seen in some of the information technology studies reviewed for this article. However, additional work on educational evaluation of misinformation videos and the tools provided by the video-sharing social media may be fruitful lines of inquiry. There were only a small portion of qualitative research available on the topic of misinformation. Research using qualitative designs is also needed because qualitative studies are helpful in exploring deep context of the spreading and consequences of misinformation, people's experiences and ways of educating students about discerning misinformation, critically evaluating information, and ways of combating misinformation. Qualitative studies might also be used to uncover the reasons underlying interest among scholars who have published or presented on the topic of misinformation. 
A particular prominent theme in misinformation literature presented lists of general cognitive experimental designs and reading strategies techniques, while others described content-specific applications of misinformation in the classroom. There is only a small portion of articles discussing online education and misinformation from a field of education perspective. Less than 10 articles discuss specifically about intersections of future education about misinformation. In the education field, the negative impact of misinformation and disinformation is unpreceded concerning educators. Some educators consider that misinformation refers to "false information shared by a source who intends to inform, but is unaware that the information is false, such as when an educator who recommends the use of a learning strategy that is not actually beneficial" ([30], p. 2). In contrast, disinformation is "false information shared by a source who has the intent to deceive and is aware that the information is false, such as when a politician claim that high-stakes testing will fix K-12 education when in fact there is no evidence to support this practice." ([30], p. 2). However, more researchers in misinformation literature reviewed use the term misinformation refers to both these two types of false information.

To this end, online education researchers focus on misinformation are calling for explorations in the future, especially the intersections of misinformation, online education, information learning, and social media. Here, YouTube learning and combating with misinformation could be a potential important research topic to explore. One particular important area that lack of studying misinformation is video aspect of misinformation and online education, despite the importance and popularity of video learning and the consequence of spreading misinformation on video media such as YouTube.

As misinformation became rampant with the online landscape on social media, YouTube became an important arena from education in various disciplines and K-12 settings. Misinformation has serious implications for how education should address this issue especially how should we teach children use social media in multimodal formats (other than texts on screen) such as YouTube to discern misinformation and scientific information. Despite the fact that misinformation became a serious issue for reading printed texts and multimedia texts, the studies on misinformation in various screens are still scare. Limited literature of misinformation mentioned YouTube and online education. It could be argued that many of the misinformation findings described in Table 1 (Methodologies Represented in the Sample of 174 Articles and Papers) would apply into many learning situations of face-to-face, hybrid, or online learning environments.

As much influential as one could imagine with the complex and comprehensible features of online learning with videos [24, 25], misinformation is a topic that has not been much explored in educational perspectives. Some conference paper has also provided possible ways of build automatic models using linguistic, acoustic, and engagement features for 200 videos' annotations for helping detecting misinformation [23]. A decade ago, Pew Internet \& American Life had already stated that majority (69\%) of U.S. internet users watch or download video online and 14\% have posted videos [44]. Now, probably the percentage is even more. YouTube is the second most popular website among all the websites available [20]. Every single day, there are 1 billion videos being watched on YouTube. In addition, video social media such as TikTok also came to prominence in people's daily sharing of videos [6]. The growth of educational video runs concurrent with broader trends in educational video viewership [41].

There is a gap between evidence-based research on how educators could be using YouTube as an effective educational tool to help students critically evaluate content knowledge, and common practices adopted by K-12 educators [28]. Given that the content on YouTube can be uploaded by anyone, educating students to combat misinformation on YouTube is a critical aspect if using YouTube as an educational tool. Future work in this line could be devoted toward information literacy and evaluation of YouTube video 
content. For example, how to identify videos containing mistakes? How to teach important evaluation and critical thinking skills when viewing videos on YouTube? One of the instructional methodologies described in the literature was video analysis [21]. With the continual growth of online education, it might become increasingly important to attain video evaluation and video literacy skills, yet more research is needed to identify the qualities of good educational video clips. Many educational videos are short productions of less than 15 minutes. Research on optimal methods of chunking content for video clips may be a valuable and timely area of study.

Admittedly, this review has some limitations. First, with the growing number of misinformation studies, between the time this review is written and publishing, there are new research that this review possibly did not catch. Second, there may be alternative studies did not use misinformation as their key words in the title, and online education in their abstract, which future systematic review could consider in offering a discussion.

\section{Conclusions}

In summary, this systematic review unveils the work on misinformation which featured various disciplines with foci on social science and health fields. In some degrees, it is remarkable that misinformation has gained the level of wide explorations and attention. The body of misinformation-related research described in this article indicates the rising interest in the topic of online education specific to disciplines. How that interest will evolve is unknown, but there is potential for future research and exploration of educational possibilities for controlling, combating, and critically evaluating misinformation.

Misinformation scholarship on various disciplines also demonstrated a growing gap for online education. Misinformation tends to be accepted by people if the misinformation is coherent, consistent with the audience's worldview, and delivered by credible source. Combating misinformation, scholars recommend strategies to deal with online education. These strategies include, for example, (1) creating team work among professional disciplines for credible resources [40,50], (2) delivering diverse professional opinions for accurate information dissemination [26], (3) updating regularly by authoritative agencies about scientific information via support of social media [50], (4) helping people to critically evaluate information using emotional and community support [53], (5) offering informational literacy education with affective support [26; 40], (6) recognizing personal needs in evaluating sources [2], (7) using detective models and regulative devices on social media to ensure publishing only scientific information for public good (e.g., flag suspicious information to warn people, [32]), and (8) promoting equitable society to help eliminate misinformation which disproportionally impacted the marginalized minorities due to race, gender, class, ethnicity, and socioeconomic statuses [14].

Lastly, while it is important to correct and combat misinformation in communication methods, social media plays the most critical roles to educate people $[3,53]$. In comparison to traditional media, online media platforms facilitate misinformation even more widely. Future studies demonstrating the role of new media in misinformation and its potential consequences are needed. Much more work is needed in terms of using an educator's perspective to approach misinformation online. Given video is now a common online media and the various online videos are available for online education, misinformation on multimodal formats such as videos deserve future integration.

Author Contributions: The author is solely responsible for the research and writing.

Funding: There is no funding for this research

Data Availability Statement: Data was supported by Scopus. 
Acknowledgments: The author thanks the editor and reviewers for your efforts in publishing this article

Conflicts of Interest: No conflict of interests.

\section{References}

1. Abhishek, A. (2021). Overlooking the political economy in the research on propaganda. Harvard Kennedy School Misinformation Review.

2. Ali, S., Khalid, A., \& Zahid, E. (2021). Is CoViD-19 immune to misinformation? A brief overview. Asian Bioethics Review, 1-23.

3. Allcott, H., Gentzkow, M., \& Yu, C. (2019). Trends in the diffusion of misinformation on social media. Research \& Politics, 6(2), 2053168019848554.

4. Austin, E. W., Borah, P., \& Domgaard, S. (2021). COVID-19 disinformation and political engagement among communities of color: The role of media literacy. The Harvard Kennedy School Misinformation Review.

5. Bapaye, J. A., \& Bapaye, H. A. (2021). Demographic Factors Influencing the Impact of Coronavirus-Related Misinformation on WhatsApp: Cross-sectional Questionnaire Study. JMIR Public Health and Surveillance, 7(1), e19858.

6. Basch, C. H., Meleo-Erwin, Z., Fera, J., Jaime, C., \& Basch, C. E. (2021). A global pandemic in the time of viral memes: COVID-19 vaccine misinformation and disinformation on TikTok. Human Vaccines \& Immunotherapeutics, 1-5.

7. Bautista, J. R., Zhang, Y., \& Gwizdka, J. (2021). Healthcare professionals' acts of correcting health misinformation on social media. International Journal of Medical Informatics, 148, 104375.

8. Bode, L., \& Vraga, E. K. (2015). In related news, that was wrong: The correction of misinformation through related stories functionality in social media. Journal of Communication, 65(4), 619-638.

9. Breakstone, J., Smith, M., Connors, P., Ortega, T., Kerr, D., \& Wineburg, S. (2021). Lateral reading: College students learn to critically evaluate internet sources in an online course. Harvard Kennedy School (HKS) Misinformation Review, $2(1)$.

10. Calo, W. A., Gilkey, M. B., Shah, P. D., Dyer, A. M., Margolis, M. A., Dailey, S. A., \& Brewer, N. T. (2021). Misinformation and other elements in HPV vaccine tweets: an experimental comparison. Journal of Behavioral Medicine, 1-10.

11. Chang, Y.K., Literat, I., Price, C., Eisman, J.I., Chapman, A., Gardner, J., \& Truss, A. (2020). News literacy education in a polarized political climate: How games can teach youth to spot misinformation. The Harvard Kennedy School (HKS) Misinformation Review, Volume 1, Issue 4

12. Coiro, J., Knobel, M., Lankshear, C., \& Leu, D. J. (Eds.). (2014). Handbook of research on new literacies. Routledge. 
13. Cook, J. (2019). Understanding and countering misinformation about climate change. Handbook of research on deception, fake news, and misinformation online, 281-306. https://www.climatechangecommunication.org/wp-content/uploads/2019/06/Cook_2019_climate_misinformation-1.pdf

14. Cummings, D., Anthony, M., Watson, C., Watson, A., \& Boone, S. (2021, March). Combating Social Injustice and Misinformation to Engage Minority Youth in Computing Sciences. In Proceedings of the 52nd ACM Technical Symposium on Computer Science Education (pp. 1006-1012).

15. Del Vicario, M., Bessi, A., Zollo, F., Petroni, F., Scala, A., Caldarelli, G.,. . \& Quattrociocchi, W. (2015). The spreading of misinformation online. Proceedings of the National Academy of Sciences of the United States of America, 113, 554559.

16. Diakidoy, I. A. N., Kendeou, P., \& Ioannides, C. (2003). Reading about energy: The effects of text structure in science learning and conceptual change. Contemporary Educational Psychology, 28(3), 335-356.

17. diSessa, A. A. (1993). Toward an epistemology of physics. Cognition and Instruction, 10, 105-225.

18. Danielson, R. W., Sinatra, G. M., \& Kendeou, P. (2016). Augmenting the refutation text effect with analogies and graphics. Discourse Processes, 53(5-6), 392-414.

19. Ecker, U. K., Lewandowsky, S., \& Tang, D. T. (2010). Explicit warnings reduce but do not eliminate the continued influence of misinformation. Memory \& cognition, 38(8), 1087-1100.

20. Freshysites. (2021). https://freshysites.com/web-design-development/most-popular-websites/

21. Goobie, G. C., Guler, S. A., Johannson, K. A., Fisher, J. H., \& Ryerson, C. J. (2019). YouTube videos as a source of misinformation on idiopathic pulmonary fibrosis. Annals of the American Thoracic Society, 16(5), 572-579.

22. Hinze, S. R., Slaten, D. G., Horton, W. S., Jenkins, R., \& Rapp, D. N. (2014). Pilgrims sailing the Titanic: Plausibility effects on memory for misinformation. Memory \& Cognition, 42(2), 305-324.

23. Hou, R., Pérez-Rosas, V., Loeb, S., \& Mihalcea, R. (2019, October). Towards automatic detection of misinformation in online medical videos. In 2019 International conference on multimodal interaction (pp. 235-243).

24. Author (2017). Learners' experiences with informally learning Chinese from watching online videos. Doctoral Dissertation. University of Rochester.

25. Author, \& Lammers, J. C. (2018). A qualitative inquiry of video learning by Chinese L2 learners: Learning internalization, understanding social realities, and reflecting on challenges. Chinese as a Second Language. The journal of the Chinese Language Teachers Association, USA, 53(3), 257-294.

26. Jaeger, P. T., \& Taylor, N. G. (2021). Arsenals of Lifelong Information Literacy: Educating Users to Navigate Political and Current Events Information in World of Ever-Evolving Misinformation. The Library Quarterly, 91(1), 19-31. 
27. Johnson, D. (2014). Reading, writing, and literacy 2.0. Teachers College Press.

28. Jones, T., \& Cuthrell, K. (2011). YouTube: Educational potentials and pitfalls. Computers in the Schools, 28(1), 75-85.

29. Kahne, J., \& Bowyer, B. (2017). Educating for democracy in a partisan age: Confronting the challenges of motivated reasoning and misinformation. American Educational Research Journal, 54(1), 3-34.

30. Kendeou, P, Robinson, D.H., \& McCrudden, M. T. (2019). Misinformation and fake news. Information Age Publishing, INC.

31. Krishna, A., \& Thompson, T. L. (2021). Misinformation about health: A review of health communication and misinformation scholarship. American behavioral scientist, 65(2), 316-332.

32. Lanius, C., Weber, R., \& MacKenzie, W. I. (2021). Use of bot and content flags to limit the spread of misinformation among social networks: a behavior and attitude survey. Social Network Analysis and Mining, 11(1), 1-15.

33. Lewandowsky, S., Ecker, U. K. H., Seifert, C. M., Schwarz, N., \& Cook, J. (2012). Misinformation and its correction: Continued influence and successful debiasing. Psychological Science in the Public Interest, 13(3), 106-131. doi:10.1177/1529100612451018

34. Lewandowsky, S., Ecker, U. K., \& Cook, J. (2017). Beyond misinformation: Understanding and coping with the "posttruth" era. Journal of applied research in memory and cognition, 6(4), 353-369.

35. Limon, M. (2002). Conceptual change in history. In M. Limon, \& L. Mason (Eds.), Reconsidering conceptual change: Issues in theory and practice (pp. 259-289). Springer, Dordrecht

36. Lombardi, D., Danielson, R. W., \& Young, N. (2016). A plausible connection: Models examining the relations between evaluation, plausibility, and the refutation text effect. Learning and Instruction, 44, 74-86.

37. Marsh, E. J., Meade, M. L., \& Roediger, H. L. (2003). Learning facts from fiction. Journal of Memory and Language, 49, 519-536.

38. Mena, P., Barbe, D., \& Chan-Olmsted, S. (2020). Misinformation on Instagram: The Impact of Trusted Endorsements on Message Credibility. Social Media+ Society, 6(2), 2056305120935102.

39. National Governors Association Center for Best Practices \& Council of Chief State School Officers. (2010). Common Core State Standards for English language arts and literacy in history/social studies, science, and technical subjects. Washington, DC.

40. Nygren, T., Folkeryd, J. W., Liberg, C., \& Guath, M. (2020, October). Students Assessing Digital News and Misinformation. In Multidisciplinary International Symposium on Disinformation in Open Online Media (pp. 63-79). Springer. 
41. Omnicorxeagency (2021). https://www.omnicoreagency.com/youtube-statistics/

42. Pan, W., Liu, D., \& Fang, J. (2021). An examination of factors contributing to the acceptance of online health misinformation. Frontiers in Psychology, 12, 524.

43. Pourghomi, P., Safieddine, F., Masri, W., \& Dordevic, M. (2017, May). How to stop spread of misinformation on social media: Facebook plans vs. right-click authenticate approach. In 2017 International Conference on Engineering \& MIS (ICEMIS) (pp. 1-8). IEEE.

44. Purcell, K. (2010). The state of online video. Washington, DC: Pew Internet \& American Life Project.

45. Rapp, D. N., \& Donovan, A. M. (2017). Routine processes of cognition result in routine influences of inaccurate content. Journal of Applied Research in Memory and Cognition, 6, 409-413.

46. Rapp, D. N., \& Braasch, J. L. G. (Eds.). (2014). Processing inaccurate information: Theoretical and applied perspectives from cognitive science and the educational sciences. Cambridge, MA: MIT Press.

47. Rojecki, A., \& Meraz, S. (2016). Rumors and factitious informational blends: The role of the web in speculative politics. New Media \& Society, 18(1), 25-43.doi:10.1177/1461444814535724

48. Sallam, M., Dababseh, D., Yaseen, A., Al-Haidar, A., Taim, D., Eid, H., ... \& Mahafzah, A. (2020). COVID-19 misinformation: Mere harmless delusions or much more? A knowledge and attitude cross-sectional study among the general public residing in Jordan. PloS one, 15(12), e0243264.

49. Spanos, K. E., Kraschnewski, J. L., Moss, J. L., Wong, A., \& Calo, W. A. (2021). Parent support for social media standards combatting vaccine misinformation. Vaccine, 39(9), 1364-1369.

50. Suarez-Lledo, V., \& Alvarez-Galvez, J. (2021). Prevalence of health misinformation on social media: systematic review. Journal of medical Internet research, 23(1), e17187.

51. Tauber, S. K., Dunlosky, J., Rawson, K. A., Rhodes, M. G., \& Sitzman, D. M. (2013). General knowledge norms: Updated and expanded from Nelson and Narens (1980) norms. Behavioral Research Methods, 45, 1115-1143.

52. Vraga, E., Tully, M., \& Bode, L. (2021). Assessing the relative merits of news literacy and corrections in responding to misinformation on Twitter. New Media \& Society, 1461444821998691.

53. Wang, R., He, Y., Xu, J., \& Zhang, H. (2020). Fake news or bad news? Toward an emotion-driven cognitive dissonance model of misinformation diffusion. Asian Journal of Communication, 30(5), 317-342.

54. Wang, Y., McKee, M., Torbica, A., \& Stuckler, D. (2019). Systematic literature review on the spread of health-related misinformation on social media. Social Science \& Medicine, 240, 112552. 
55. Wilner, T., \& Holton, A. (2020). Breast Cancer Prevention and Treatment: Misinformation on Pinterest, 2018. American Journal of Public Health, 110(S3), S300-S304.

56. Wolf, M. S., Davis, T. C., Shrank, W., Rapp, D. N., Bass, P. F., Connor, U. M., ... \& Parker, R. M. (2007). To err is human: patient misinterpretations of prescription drug label instructions. Patient education and counseling, 67(3), 293-300. 